\title{
Dynamic light scattering during shear: measurements of diffusion coefficients
}

\author{
D. Rusu $^{\mathrm{a}}$, D. Genoe ${ }^{\mathrm{a}}$, P. van Puyvelde ${ }^{1, \mathrm{a}}$, E. Peuvrel-Disdier ${ }^{\mathrm{a}, *}$, P. Navard ${ }^{\mathrm{a}}$, G.G. Fuller $^{\mathrm{b}}$ \\ ${ }^{a}$ Ecole des Mines de Paris, Centre de Mise en Forme des Matériaux, UMR CNRS 7635, BP 207, 06904 Sophia-Antipolis, France \\ ${ }^{\mathrm{b}}$ Chemical Engineering Department, Stanford University, Stanford, CA 94305-5025, USA
}

Received 17 February 1998; revised 15 April 1998; accepted 6 May 1998

\begin{abstract}
A method for determining diffusion coefficients during shear by dynamic light scattering is presented. The direct determination of diffusion coefficient during flow is hampered by the domination of the convection terms over the diffusion terms except in a very narrow azimuthal scattering angle range that is impossible to reach experimentally. The method described here offers the advantage to this constraint. The technique is based on a double measurement of homodyne spectrum of light scattered from small scatterers immersed in a medium subjected to a simple shear flow. This can be applied to polymer solutions. Verification is provided using suspensions of polystyrene spheres in Newtonian media and a transparent rheometer. (C) 1998 Elsevier Science Ltd. All rights reserved.
\end{abstract}

Keywords: Dynamic light scattering; Shear; Diffusion coefficient

\section{Introduction}

Dynamic light scattering, also called photon correlation or quasi-elastic light scattering spectroscopy, is a noninvasive probe of diffusion in complex fluids. This technique enables the direct determination of the mutual diffusion coefficient of the scattering units [1-3]. In dilute solutions, this diffusion coefficient can be used to estimate the size of particles and, in the case of macromolecules, the molecular weight. Its usual practical application is therefore in particle sizing. Very little work has concerned the application of this technique in the presence of a flow [4-6]. Edwards et al. [4] and Pike [5] showed that the mean velocity of the scattering centers can be accessed from the heterodyne mode of the scattering light whereas Fuller et al. [6] showed that the homodyne mode leads to the determination of velocity gradients. A potential application is the determination of the transverse diffusion coefficient of polymer chains during flow. One of the possibilities is to use small Brownian spheres that can serve as probes for measuring such coefficients. The difficulty that was readilly faced by previous researchers is the near impossibility of measuring diffusion coefficients during flow since the measure is dominated by

\footnotetext{
* Corresponding author.

${ }^{1}$ Present address: Department of Chemical Engineeering, K.U. Leuven, de Croylaan 46, 3001, Leuven, Belgium.
}

the effect of flow and not the effect of diffusion. The measurement must be performed in an extremely narrow range of scattering angles that cannot be achieved experimentally.

In this article we propose a new method, based on a double measurement of the homodyne spectrum of light scattered from small particles immersed in a medium subjected to a simple shear flow, to determine diffusion coefficients during shear. By this method, the scattering angle must remain in an angular range that is easily obtained. To illustrate this method and show its validity, we will apply it to a case where the flowing material is known to have the same diffusion coefficient with and without flow. We will then measure the diffusion coefficient during flow with our new method and show that it is the same as the one at rest, as expected. Any other measurement by a direct method would have been impossible.

We will first give the theoretical basis of this new method, then describe the apparatus to perform the experiments and detail the experimental results.

\section{Theoretical approach and origin of this work}

The basic experiment considered here is the light scattered by small monodispersed spheres which are immersed in a liquid undergoing a simple shear flow. The spheres are assumed to be rigid and small enough so that their mean 
translational velocity is indistinguishable from the velocity of the suspending liquid. The concentration of particles is low so that multiple scattering events can be neglected. The size of the spheres is assumed to satisfy the RayleighDebye condition [1]. In order to simplify the development of the correlation function [6], the parameters characterizing the experiment must satisfy the fact that the transit time scale for the spheres is large compared with the shortest time scales characteristic of sphere displacement over a length scale $\mid \mathrm{q}^{-1}$, which can be expressed as:

$$
\frac{1}{\dot{\gamma} L q_{x}} \ll \frac{1}{\dot{\gamma}}
$$

where $\dot{\gamma}$ is the shear rate, $\mathbf{q}$ the scattering vector, $q_{x}, q_{y}, q_{z}$ its projections in the set of axes originated at the scattering volume ( $x$ being the axis parallel to the flow direction, $y$ being along the vorticity axis), and $L$ a characteristic length of the scattering volume.

Under these conditions, Fuller et al. [6] show that the homodyne correlation function of a suspension submitted to a shear flow can be written as the following product of two terms (Eq. (2)), a diffusive term due to the Brownian motion of the spheres in the shear flow and a convective term related to the position correlation within the flow:

$$
\begin{aligned}
F_{2}(q, t, \dot{\gamma})= & \exp \left\{-2 D_{\text {flow }}\left[q_{x}^{2}\left(1+1 / 3(\dot{\gamma} t)^{2}\right)+\dot{\gamma} t q_{x} q_{y}\right.\right. \\
& \left.\left.+q_{y}^{2}+q_{z}^{2}\right] t\right\}\left|\iiint d^{3} x I(x) \exp \left\{-i \dot{\gamma} q_{x} y t\right\}\right|^{2}
\end{aligned}
$$

where $D_{\text {flow }}$ is the diffusion coefficient of the spheres suspended in a liquid undergoing a simple shear flow and $I(x)$ the light intensity profile.

This correlation function varies on two time scales, 1/ $D_{\text {flow }} q^{2}$ and $1 / \dot{\gamma} L q_{x}$. The correlation function being dominated by the event with the shortest time scale, this equation predicts that in most cases, the strongest contribution to the correlation function arises from the mean velocity gradient in the scattering volume and that measurements of diffusion coefficients can only be performed at the azimuthal angle of $90^{\circ}$, where $q_{x} \rightarrow 0$. The azimuthal angle characterizes the angular position in the $x y$ plane (defined by the shear direction and the vorticity axis) at which the scattered intensity is analysed. $0^{\circ}$ corresponds to the $x$ axis. An estimate of the azimuthal angle required for the diffusive term to dominate the signal is given by the relation:

$$
\frac{1}{D_{\text {flow }} q^{2}} \ll \frac{1}{\dot{\gamma} L q_{x}}
$$

By taking characteristic values of our experimental conditions (see 'Validation of the method'), the contribution of random motions due to diffusion can only be determined in a very narrow angle range of $90 \pm 0.00001^{\circ}$. This is a very unrealistic condition and there is no way experiments can be performed within this range. This has always been a difficulty that was not overcome.

This weaker influence of the flow on the correlation position when approaching an azimuthal angle of $90^{\circ}$

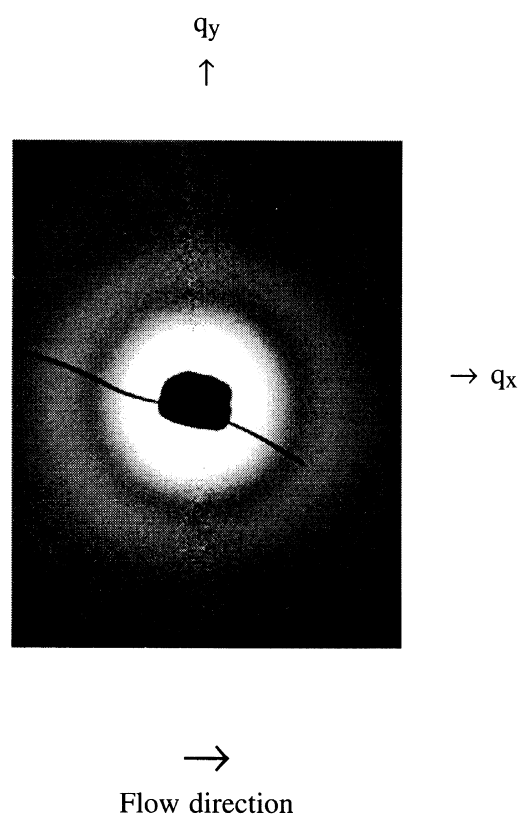

Fig. 1. Small-angle light scattering pattern observed on a suspension of monodispersed polystyrene spheres (diameter $13.7 \mu \mathrm{m}$ from Coulter Electronics, Luton, U.K.) in a solution of polyacrylamide in water subjected to a shear rate of $27 \mathrm{~s}^{-1}$.

compared with the flow direction can be observed on the light scattering pattern of a suspension of polystyrene spheres in water subjected to a shear flow (Fig. 1). The pattern obtained during flow exhibits a zone at $90^{\circ}$ compared with the flow direction where the speckle vibrates in a much slower manner than elsewhere. The influence of the azimuthal angle is more precisely illustrated in Fig. 2, where correlation functions measured during shear at different positions are compared with one determined at rest. This figure shows that even if the correlation function during flow changes drastically around $90^{\circ}$ (compare $90^{\circ}$ and $90.5^{\circ}$ ), as also observed on the scattering pattern, the determination of a diffusion coefficient during flow is implied to be exactly at $90^{\circ}$ which is impossible.

The convective term being the term with the strongest flow dependence (around $90^{\circ}$ ), this term must be eliminated to have access to the contribution of the diffusive motion. Since this term only depends on experimental conditions (light profile intensity, applied shear rate), it can be cancelled by performing two separate measurements of the correlation function in two experiments having identical conditions with respect to the applied shear rate and spheres, but using two different suspending media A and B. Giving $G(q, t, \dot{\gamma})$ as the ratio of the two correlation functions, this ratio can be simplified to:

$$
\begin{aligned}
G(q, t, \dot{\gamma})= & \exp \left\{-2\left(D_{A_{\text {flow }}}-D_{B_{\text {flow }}}\right)\left[q_{x}^{2}\left(1+1 / 3(\dot{\gamma} t)^{2}\right)\right.\right. \\
& \left.\left.+\dot{\gamma} t q_{x} q_{y}+q_{y}^{2}+q_{z}^{2}\right] t\right\}
\end{aligned}
$$

where $D_{A_{\text {flow }}}$ and $D_{B_{\text {flow }}}$ are the diffusion coefficients of the spheres suspended respectively in medium A and B and in the presence of a shear flow. 


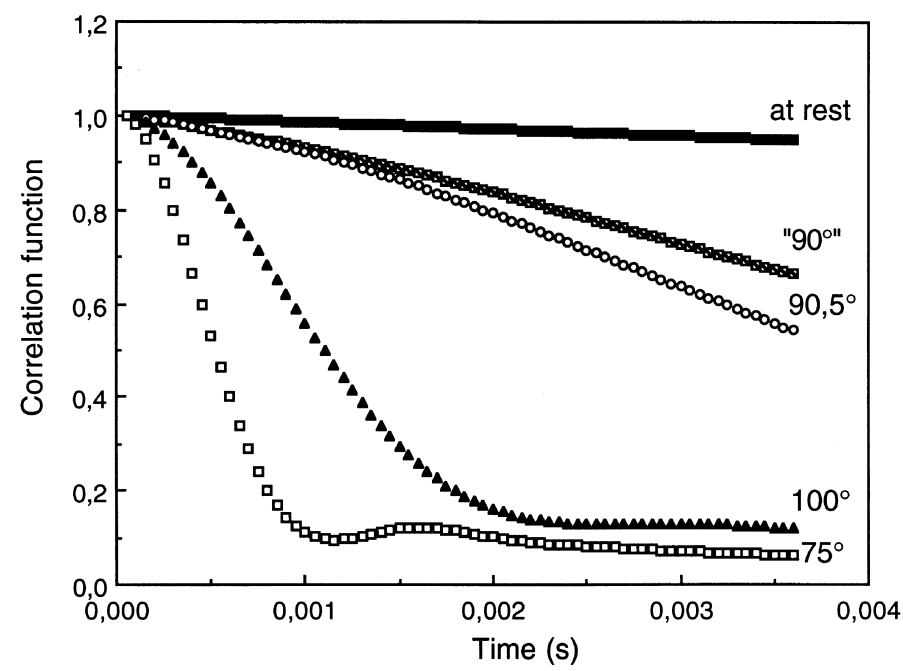

Fig. 2. Homodyne correlation functions measured on a suspension of polystyrene spheres in water subjected to a simple shear flow (21 $\left.\mathrm{s}^{-1}\right)$, at different azimuthal angles. Comparison with the correlation function measured at rest.

This can be rewritten as:

$$
\begin{aligned}
G(q, t, \dot{\gamma})= & \exp \left\{-2\left(D_{A_{\text {flow }}}-D_{B_{\text {flow }}}\right)\left[q^{2} t+\dot{\gamma} q_{x} q_{y} t^{2}\right.\right. \\
& \left.\left.+2 / 3 \dot{\gamma}^{2} q_{x}^{2} t^{3}\right]\right\}
\end{aligned}
$$

Fitting the Napierian logarithm of $G(q, t, \dot{\gamma})$ by a thirddegree polynomial on time gives access to the coefficient of the first degree monomial which is equal to:

$-2\left(D_{A_{\text {flow }}}-D_{B_{\text {flow }}}\right) q^{2}$

Choosing one of the suspending liquids as a Newtonian medium (for example B), for which the diffusion coefficient during flow $\left(D_{B_{\text {flow }}}\right)$ is equal to the one at rest $\left(D_{B_{\text {rest }}}\right)$, enables one to determine the diffusion coefficient $D_{A_{\text {flow }}}$ in the presence of a shear flow.

\section{Description of the apparatus}

A time correlation set-up to analyse the light scattered at small angle by a sample submitted to a shear flow was designed in close collaboration with SEMATech (Nice, France). This device can be used in conjunction with a transparent rheometer (described in detail elsewhere [7]). The experiment comprises a laser beam illuminating the sample contained in the shear cell and the time correlation device to analyse the scattered intensity. A schematic drawing of the global experiment is shown in Fig. 3. The light beam is a polarized $\mathrm{He}-\mathrm{Ne}$ laser that emits light with a monochromatic wavelength of $632.8 \mathrm{~nm}$ in vacuum. The sample is contained in a transparent rotating plate-andplate shear cell, the thickness of the sample being $250 \mu \mathrm{m}$. The scattered intensity is detected by a photomultiplier fixed on a bench. This bench is mounted on a mechanical part which enables rotation of the bench around the incident laser beam axis and changing the distance between the scattering elements and the detector.
Mechanical obstruction due to the fact that the device is mounted under the rheometer restricts the range of polar and azimuthal angles which can be investigated. Typically, the photomultiplier can be located at polar angles ranging from 5 to $36^{\circ}$ and azimuthal angles between -90 and $+90^{\circ}$. As the distance between the sample and the detector can be varied and as the position and orientation of the latter is fixed on the bench by construction, a particular optical system (pinhole + lens + pinhole, developed by SEMATech) has been set up in front of the photomultiplier (Fig. 4). This optical system avoids any misalignement of the detector towards the polar angle of the scattered light to be detected. The photomultiplier is connected to the SEMATech UNICOR correlator which analyzes and calculates the homodyne correlation (or auto-correlation) functions of the scattered light with sample times ranging from $50 \mathrm{~ns}$

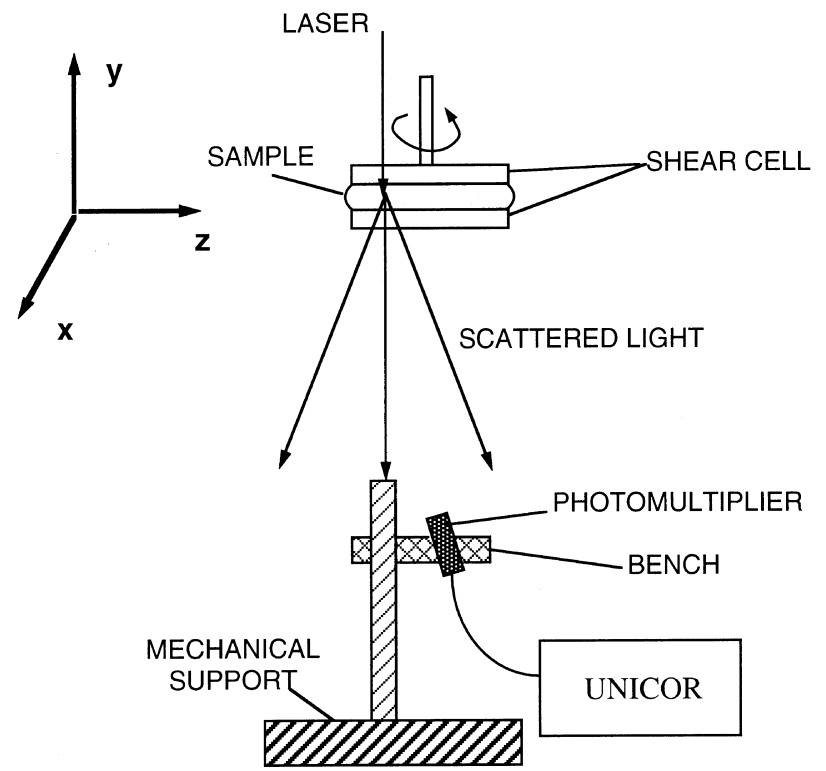

Fig. 3. Schematic drawing of the global experimental set-up. 


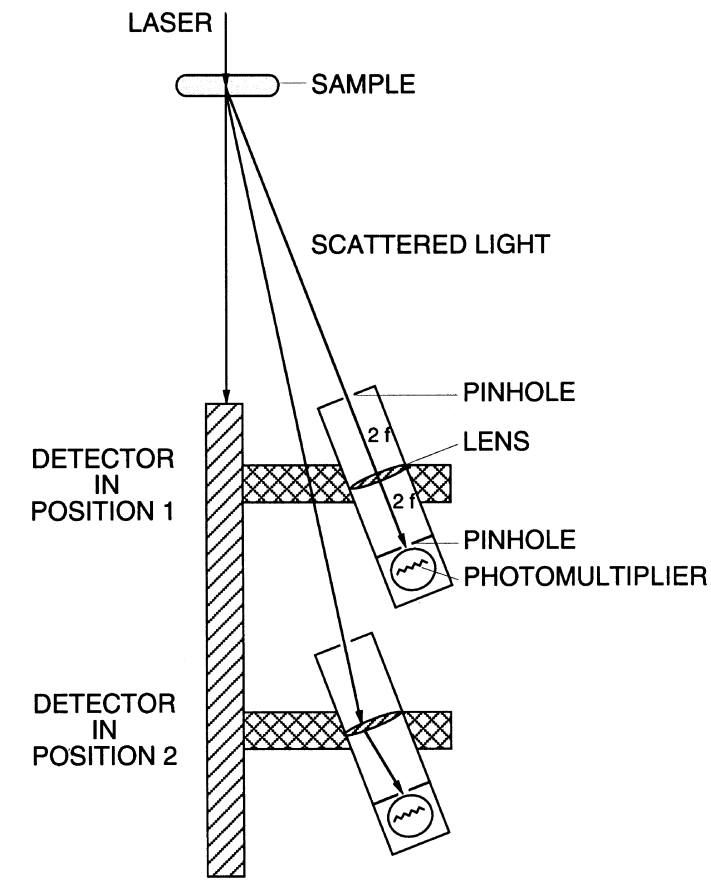

Fig. 4. Close-up of the optical system in front of the photomultiplier. Illustration of its role when the distance between the sample and the detector is changed.

to $160 \mathrm{~ms}$. This is carried out in real time. The UNICOR correlator is a digital correlator which, used in the linear mode, has four sections with 18 channels each.

\section{Validation of the method}

One way to validate the approach described above is to use a system for which the diffusion coefficient during flow is the same as the one at rest. This is the case of a suspension of monodispersed hard spheres immersed in a Newtonian medium. We will thus measure the diffusion coefficient during flow and compare it to the diffusion coefficient at rest. This will validate the proposed method.

This was performed by using suspensions of monodispersed polystyrene spheres (diameter $1.1 \mu \mathrm{m}$ from Coulter Electronics Limited, Luton, England) in two different Newtonian media, either water or a mixture of water and glycerol. The volume fraction of glycerol when mixed with water is $15 \%$. The volume fraction of spheres in the suspension is, in all cases, $0.11 \%$.

The experiments were performed at room temperature $\left(23.5^{\circ} \mathrm{C}\right)$. The auto-correlation functions at rest and during flow were measured at a polar angle of $18^{\circ}$, and in order to minimize the relative importance of the convective term at an azimuthal angle as close as possible to $90^{\circ}$, relative to the flow direction. The $90^{\circ}$ position was determined experimentally by searching for the azimuthal angle at which the correlation function measured during shear had the longest relaxation time. Sample times used for the determination

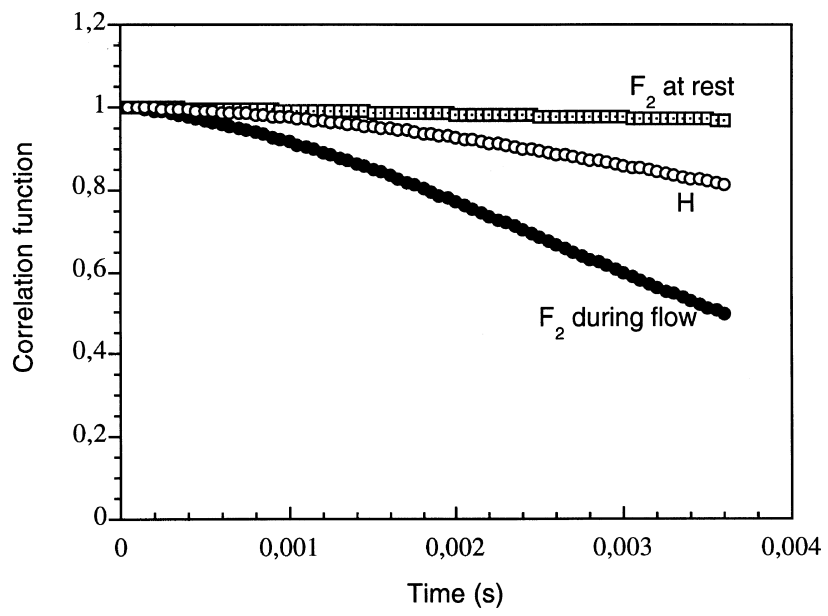

Fig. 5. Comparison between correlation functions measured (on spheres suspended in water) at rest, during shear and calculated according to Eq (4). In order to compare $G(q, t, \dot{\gamma})$ with the other functions, this function was multiplied by the correlation function measured in water at rest. This new function is noted $H$ on the graph: $H(q, t, \dot{\gamma})=G(q, t, \dot{\gamma}) \cdot \exp \left(-2 D_{B_{\text {rest }}} q^{2} t\right)$.

of the correlation functions were around $50 \mathrm{~ms}$. The applied shear rate was $21 \mathrm{~s}^{-1}$ (at the location of the investigated scattering volume).

Fig. 5 shows an example of comparisons between correlation functions measured (on spheres suspended in water) at rest, during shear and calculated according to Eq. (4). The diffusion coefficient during flow of the suspension in water is determined by the use of Eq. (4) and the measurements of different correlation functions (correlation functions during shear of the spheres in water and in the solvent mixture, and at rest of the same suspension in the mixture of water and glycerol). The determination of the diffusion coefficient of the suspension in water at rest was used for comparison with the coefficient during flow calculated according to the proposed method. The diffusion coefficients of the spheres in both media measured at rest and determined during flow using the developed approach are summarized in Table 1.

The difference between the diffusion coefficients measured at rest is directly related to the viscosity difference

Table 1

Diffusion coefficients of the polystyrene spheres suspended either in water or in the mixture of water and glycerol measured at rest or during shear (using Eq. (4))

\begin{tabular}{lll}
\hline & \multicolumn{2}{l}{ Suspending medium } \\
\cline { 2 - 3 } & Water & Water + glycerol \\
\hline$D_{\text {rest }}$ & $4.3 \times 10^{-9} \mathrm{~cm}^{2} / \mathrm{s}$ & $2.6 \times 10^{-9} \mathrm{~cm}^{2} / \mathrm{s}$ \\
$D_{A_{\text {flow }}}$ & $4.0 \times 10^{-9} \mathrm{~cm}^{2} / \mathrm{s}^{\text {a }}$ & $2.6 \times 10^{-9} \mathrm{~cm}^{2} / \mathrm{s}$ \\
\hline
\end{tabular}

${ }^{a}$ Using the solvent mixture as the reference Newtonian medium (fluid B in the developed approach) enables calculation of the diffusion coefficient during shear of the spheres suspended in water

${ }^{b}$ Respectively, the diffusion coefficient during shear of the spheres suspended in the solvent mixture is determined using water as the reference Newtonian medium 
between the two suspending media. It was checked that both values give the correct sphere diameter [1-3]. However, the main result of these measurements concerns the fact that the diffusion coefficients determined during shear are equal to the ones measured at rest, which was expected for Newtonian suspending media and thus validates the proposed approach.

\section{Conclusion}

We have presented a new method, based on a double measurement of the homodyne spectrum of light scattered from small spheres immersed in a medium subjected to a simple shear flow, to determine diffusion coefficients during shear. This approach was validated in the case of suspensions of monodispersed hard spheres immersed in Newtonian media. Applying this method to suspensions of spheres immersed in polymer solutions will be of great interest, since it will contribute information on diffusive motions in the suspending medium and thus indirectly on the conformational state of the polymer chain.

\section{Acknowledgements}

We would like to thank the CNRS for financial support and the European Community Erasmus Programme for undergraduate grants to P.G. and P.V.P. We are also grateful to SEMATech for their help in developing and setting up the device.

\section{References}

[1] Berne BJ, Pecora R. Dynamic light scattering with applications to chemistry, biology, and physics. New York: Wiley, 1976.

[2] Pecora R. Dynamic light scattering - applications of photon correlation spectroscopy. New York: Plenum, 1985.

[3] Cummins HZ, Pike ER. Photon correlation and light beating spectroscopy. New York: Plenum, 1974.

[4] Edwards RV, Angus JC, French MJ, Dunning JW. J Appl Phys 1971;42:837.

[5] Pike ER. Photon correlation velocimetry. In: Cummins HZ, Pike ER, editors. Photon correlation spectroscopy and velocimetry. New York: Plenum, 1977.

[6] Fuller GG, Rallison JM, Schmidt RL, Leal LG. J Fluid Mech 1980;100:555

[7] Riti JB, Navard P. Synth Polym J 1995;1(2-3):1. 